\title{
Repair Effects of Bone Marrow Mesenchymal Stem Cells on Demyelination of Trigeminal Ganglion in Rats with Trigeminal Neuralgia
}

\author{
Xuebin Yan', Yi Liu², Shanzi Yu', Dong Huang', Rong Hu' \\ 'Department of Pain, The Third Xiangya Hospital of Central South University, Changsha, People's Republic of China; ${ }^{2}$ Department of Anesthesia, The \\ First Hospital of Changsha, Changsha, People's Republic of China
}

Correspondence: Rong Hu, Department of Pain, The Third Xiangya Hospital of Central South University, Changsha, People's Republic of China, Tel+8618973 I62969, Email vroro@|26.com

Objective: The current study investigated the effects of bone marrow mesenchymal stem cells (BMSCs) on pain behavior in rats with trigeminal neuralgia induced by infraorbital nerve chronic constriction injury (ION-CCI), and the repair effects of BMSCs on pathological changes in trigeminal ganglion demyelination.

Methods: BMSCs or phosphate-buffered saline (PBS) alone were injected around trigeminal ganglion in ION-CCI rats via a rat brain stereotaxic apparatus. Mechanical pain threshold (von Frey test) and face grooming behavior were measured in each group. Recovery of demyelination of trigeminal ganglion was observed via electron microscopy 2 weeks later, and BMSC differentiation was observed via immunofluorescence.

Results: Rats in the BMSC group exhibited significant improvements in mechanical pain threshold and face grooming behavior compared with the PBS group. BMSCs could repair demyelinating changes in trigeminal ganglion in ION-CCI rats. Only cells expressing GFAP, S-100, and p75 were observed via immunofluorescence, and no PKH67-labeled BMSCs were observed in the trigeminal ganglion. No BMSC differentiation was observed in the trigeminal ganglion.

Conclusion: Injection of BMSCs around the trigeminal ganglion could relieve trigeminal neuralgia effectively and repair trigeminal ganglion demyelination. No differentiation of BMSCs injected around the trigeminal ganglion into Schwann cells was observed. The mechanism of trigeminal neuralgia demyelination repair requires further investigation.

Keywords: bone marrow mesenchymal stem cells, demyelination, trigeminal neuralgia, treatment

\section{Introduction}

Trigeminal neuralgia (TN) is a common craniofacial pain disease that causes severe and recurrent pain, and currently there is no ideal treatment. It entails a type of neuropathic pain, and its main pathophysiological basis is demyelination of peripheral branches of the trigeminal nerve and trigeminal ganglion. ${ }^{1-5}$ It is generally believed that the demyelination negatively affects the insulating function of the myelin sheath, causing heterotopic impulses and contact transmission between neurons. ${ }^{4,5}$

Consistent with the important role of Schwann cells in the process of peripheral nerve myelination and regeneration, Schwann cell transplantation can repair trigeminal nerve demyelination. ${ }^{6}$ The source of Schwann cells greatly limits their widespread clinical application, however, and they are highly immunogenic. ${ }^{7,8}$ Bone marrow mesenchymal stem cells (BMSCs) are a major type of pluripotent mesenchymal stem cells that have the potential to differentiate into a variety of cell types. They have a wide range of sources, are easy to isolate and expand in vitro, have immunomodulatory properties, and can secrete a variety of nutritive mediators. ${ }^{9}$ They are also well tolerated and have no adverse side effects when directly injected. ${ }^{10}$ Animal experiments and clinical studies have shown that BMSCs can effectively relieve orofacial pain caused by systemic intravenous or local injury. ${ }^{11,12}$ Many studies have shown that BMSCs can promote 
remyelination, but the mechanisms involved remain unclear. ${ }^{11-13}$ Wang et al ${ }^{14}$ reported that specific factors in the nerve lesion microenvironment could induce the differentiation of transplanted BMSCs into functional Schwann-like cells.

The current study investigated whether BMSCs could be used to treat TN by repairing trigeminal nerve demyelination, and whether the repair mechanisms involved included BMSC differentiation. An infraorbital nerve chronic constriction injury (ION-CCI) model was used to investigate repairing effects of BMSCs on rat trigeminal ganglion demyelination, and whether BMSCs could differentiate into Schwann cells in vivo.

\section{Materials and Methods}

\section{Animals and Groups}

Twenty-eight specific pathogen-free-grade adult male Sprague-Dawley rats weighing 180-220 g were raised at the Department of Laboratory Animals of Central South University at a constant temperature of approximately $24^{\circ} \mathrm{C}$, under a $12 \mathrm{~h} / 12 \mathrm{~h}$ circadian rhythm, and with food and water available ad libitum. Prior to the experiments, the rats were acclimatized for 3 days to reduce the influence of environmental factors. They were then subjected to a week of adaptive training for behavior tests, to identify and exclude rats with excessively strong stress responses such as excessive agitation or becoming too quiet.

Rats were randomly divided into four groups ( $\mathrm{n}=7$ ); a sham group (sham operation), an ION-CCI group (TN model), a BMSC group (ION-CCI + BMSCs), and a phosphate-buffered saline (PBS) group (ION-CCI + PBS). All rats were purchased from the Department of Laboratory Animals of Central South University, and all experimental procedures were approved by the Animal Management and Use Committee of the Department of Laboratory Animals of Central South University (approval number 2018sydw0209) and were carried out in strict accordance with the recommendations in the Guide for the Care and Use of Laboratory Animals of the National Institutes of Health.

\section{Cell Preparation and Staining}

Sprague Dawley rat BMSCs (Cyagen Biosciences, Guangzhou, China, Art.No.RASMX-01001) were inoculated into complete Sprague Dawley rat BMSC medium (Cyagen Biosciences, Art.No.RASMX-90011) then cultured in an incubator at $37^{\circ} \mathrm{C}$ and $5 \% \mathrm{CO}_{2}$. After $48 \mathrm{~h}$, the cells were put into fresh complete medium, which was replaced with fresh complete medium every 2 days. When the cells reached 80-90\% confluence they were stained with PKH67 green fluorescence (BaiAoLaiBo Technology, Beijing, China) then resuspended in PBS at $10^{7} / \mathrm{mL}$ prior to use.

\section{Preparation of TN Model}

In accordance with the steps described by Kernisant et al, ${ }^{15}$ after the rats were anesthetized via $1 \%$ pentobarbital sodium injection into the cavum abdominis $(40 \mathrm{mg} / \mathrm{kg}$ ) the hair above their left eye was shaved. A longitudinal skin incision of approximately $7 \mathrm{~mm}$ was then made $2 \mathrm{~mm}$ above the left eye along the curve of the frontal bone. The periosteum and muscle were carefully separated along the upper rim of the orbit, and the orbital contents were gently pulled to one side to expose the infraorbital nerve stuck to the maxilla, approximately $8 \mathrm{~mm}$ deep from the orbital rim. An approximately 5-mm length of the infraorbital nerve was gently dissociated from the connective tissue. Two chromium catgut wires were then passed under the infraorbital nerve and ligated loosely to the extent that the diameter of the nerve after ligation was slightly thinner than it had been, as observed via microscopy. The nerve was not completely blocked, and blood circulation remained. The distance between the two ligation lines was $2 \mathrm{~mm}$. Lastly, the surgical incision was sutured and disinfected. Rats in the sham group only had the nerve exposed, without ligation.

\section{Intervention}

According to the procedure described by Yang et al, ${ }^{16}$ after anesthetization via $1 \%$ pentobarbital sodium injection into the cavum abdominis $(40 \mathrm{mg} / \mathrm{kg}$ ) rats were mounted onto a brain stereotaxic apparatus, and the micro-syringe positioner on the stereotaxic device was placed at $3.4 \mathrm{~mm}$ posterior to the bregma, $3.5 \mathrm{~mm}$ left of the midline, and $6.0 \mathrm{~mm}$ ventral from the surface of the skull. Twenty microliters of a PBS suspension containing BMSC was slowly injected through the micro syringe at a rate of $4 \mu \mathrm{L} / \mathrm{min}$. After the injection, the micro syringe was kept at the needle insertion point for $10 \mathrm{~min}$, then 
slowly withdrawn. Lastly, the surgical incision was sutured and disinfected. Rats in the PBS group were injected with the same amount of PBS via the stereotaxic device.

\section{Observation Index and Method}

\section{Pain Behavior Test}

Mechanical pain thresholds were measured via the von Frey test on the day before modeling and 1, 3, 7, 10, 12, 16, 20, and 24 days after modeling (intervention was on the 9th day after modeling). Spontaneous pain was measured by observing face grooming behavior, which was recorded in a 30-min video. Before the test, the rats were acclimatized to the test environment for at least $1 \mathrm{~h}$ until they were calm.

\section{Trigeminal Ganglion Demyelination}

The trigeminal ganglion of the rats in the ION-CCI and sham groups were collected on the 16th day after modeling. After deep anesthesia with $1 \%$ pentobarbital sodium $(40 \mathrm{mg} / \mathrm{kg})$ the rats were fixed via $4 \%$ paraformaldehyde perfusion. They were then decapitated, and approximately $2-3 \mathrm{~mm}$ of the injured trigeminal ganglion was quickly removed and placed in 2.5\% glutaraldehyde fixative solution. Lastly, they were sent to the electron microscope room of Xiangya Hospital, Central South University for sectioning and observation. The rats in the BMSC and PBS groups underwent the same operation on the 24th day after modeling (the 15th day after stereotaxic injection).

\section{Immunofluorescence Staining}

The trigeminal ganglion of rats in the BMSC group were collected on the 24th day after modeling (the 15th day after the stereotaxic injection). After being fixed and dehydrated, the trigeminal ganglion was embedded in Tissue-Tek O.C.T. Compound and divided into sections measuring approximately $6 \mu \mathrm{m}$ for immunofluorescence staining. The primary antibodies (Abcam, UK) used were rabbit anti-GFAP (1:200), rabbit anti-S100 (1:200), and rabbit anti-P75 (1:200), and the secondary antibody (Jackson ImmunoResearch, USA) used was Cy3-conjugated goat anti-rabbit IgG(H+L) (1:200). Lastly, DAPI solution was used to stain the nucleus, and the samples were observed via fluorescence microscopy.

\section{Statistical Analyses}

Two-way repeated-measures analysis of variance was used to compare the means, and $p<0.05$ was considered statistically significant.

\section{Results}

\section{BMSCs Were Successfully Labeled by PKH67 in vitro}

BMSCs were grown to adherence under standard culture conditions for approximately $24 \mathrm{~h}$. Under an inverted microscope, the cells appeared spindle-shaped with bright cytoplasm and clear nuclei, and they were growing spirally. On the 2nd day after PKH67 staining the cells had grown well and were uniformly stained. They emitted strong green fluorescence under fluorescence microscopy. On the 14th day after staining the green fluorescence intensity had decreased with cell proliferation, but it was still clearly visible (Figures 1 and Figure 2).

\section{BMSCs Were Successfully Injected Around the Ipsilateral Trigeminal Ganglion}

BMSCs with PKH67 were injected around the left trigeminal ganglion of ION-CCI rats via a rat brain stereotaxic apparatus. The trigeminal ganglion were collected on the 2nd day after the stereotaxic injection. Under a fluorescence microscope most of the green fluorescence was tightly wrapped around the trigeminal ganglion, and only a small amount of green fluorescence was in the needle tract of trigeminal ganglion (Figure 3).

\section{TN Could Be Relieved by Injecting BMSCs Around Ipsilateral Trigeminal Ganglion}

In von Frey tests, the mechanical pain thresholds of the two groups began to differ significantly on the 7th day after modeling $(p>0.05)$, and the threshold of the ION-CCI group was significantly lower than that of the sham group. On the 16th day after modeling, the ION-CCI group reached the lowest pain threshold, which differed significantly from that of 

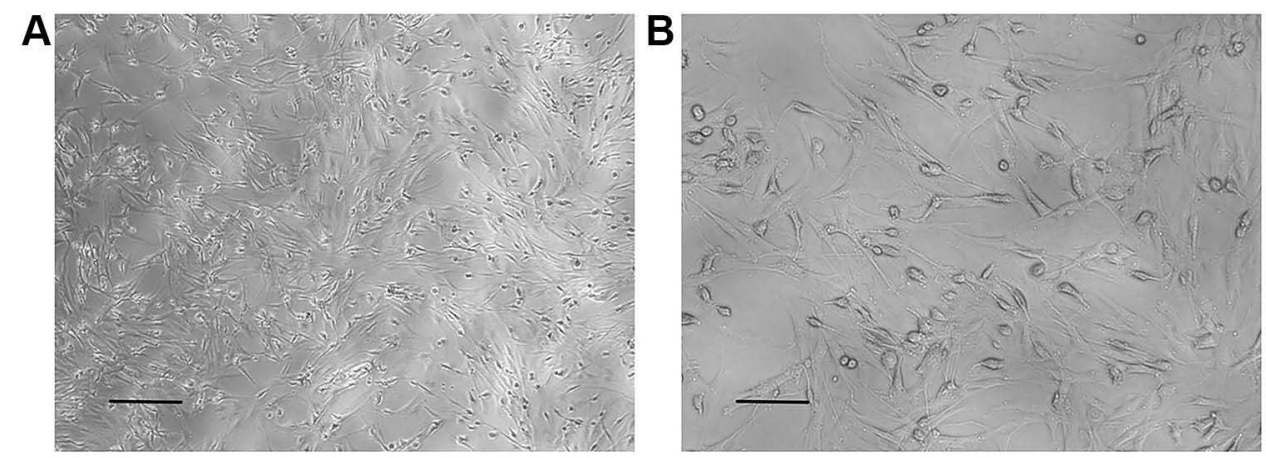

Figure I BMSCs observed under a light microscope after resuscitation. $(\mathbf{A}) \times 10$ multiple eyepiece, $\times 10$ multiple objective. Scale bar $=0.2 \mathrm{~mm}$. $($ B $) \times 10$ multiple eyepiece, x20 multiple objective. Scale bar $=0.1 \mathrm{~mm}$.
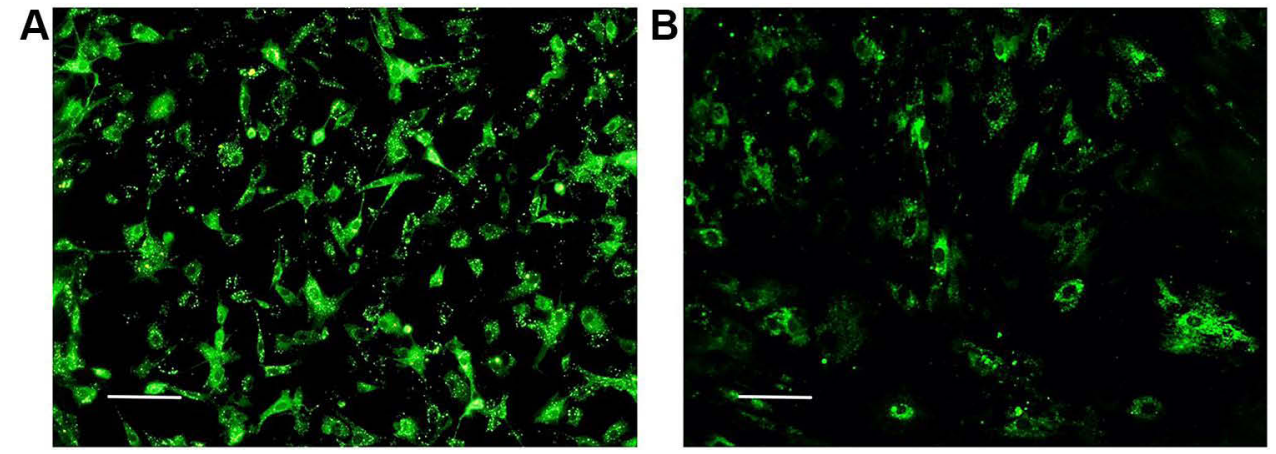

Figure 2 BMSCs observed under a fluorescence microscope after PKH67 staining. (A) Second day after staining. (B) Fifteenth day after staining. Scale bar = 0.1 mm.
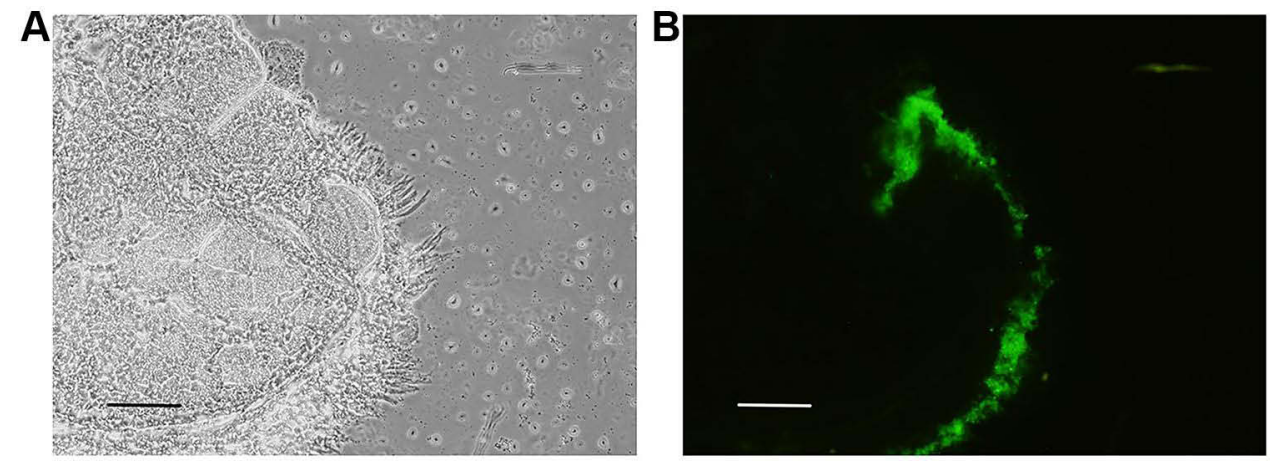

Figure 3 Distribution of PKH67-labeled BMSCs in the ipsilateral TG on the 2nd day after stereotaxic injection. (A) Trigeminal ganglion observed under a light microscope. (B) Trigeminal ganglion observed under a fluorescence microscope. Scale bar $=0.2 \mathrm{~mm}$.

the sham group $(p<0.01)$. In the video behavior tests, the face grooming time of the two groups after modeling increased compared with that before modeling. The face grooming time of the sham group began to decrease on the 3rd day, whereas that of the ION-CCI group showed no significant decrease and was generally longer than that of the sham group $(p<0.05)$ (Figure 4A and B).

After BMSC intervention, the mechanical pain threshold of the ION-CCI rats increased, and face grooming time decreased. In von Frey tests, there was no difference in mechanical pain thresholds between the two groups after modeling $(p>0.05)$. After the intervention, the pain threshold of the BMSC group gradually recovered on the 12th day after modeling, and it had recovered to baseline level (pain threshold before modeling) on the 16th day after modeling $(p>0.05)$. The PBS group continued to decline, reaching the lowest point on the 16th day after modeling, and it differed 
A

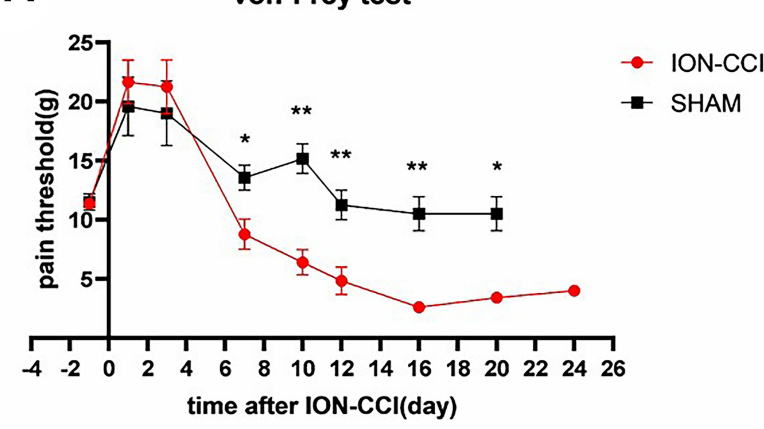

C

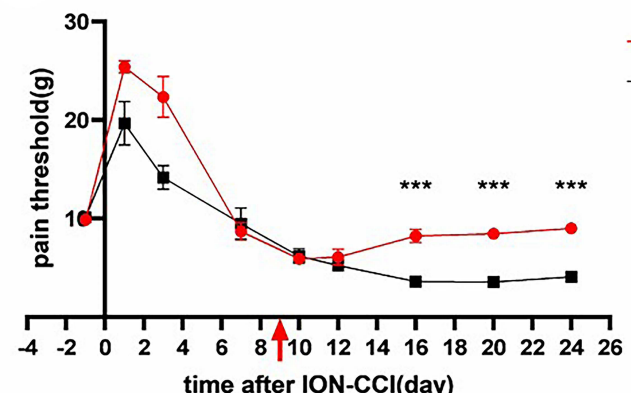

B

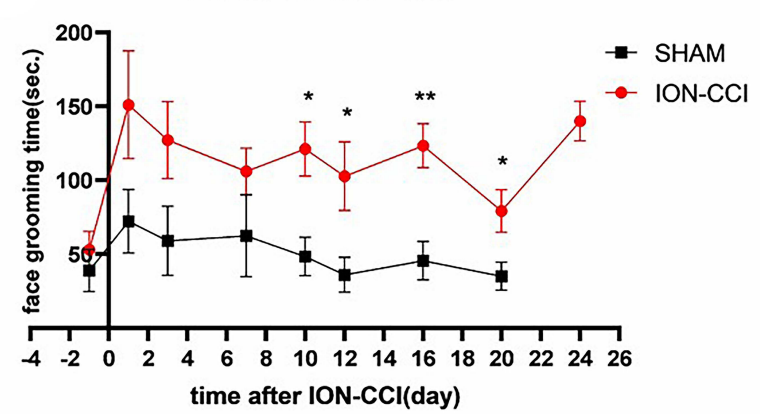

D

video behavior test

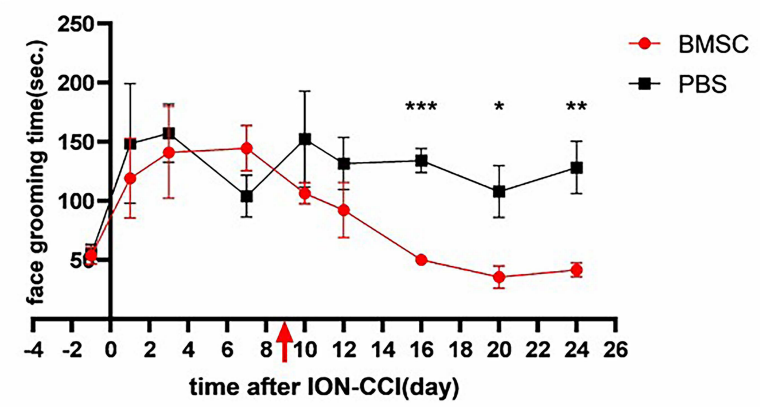

Figure 4 Effect of BMSC transplantation on trigeminal neuralgia. (A) The mechanical pain threshold was significantly decreased in ION-CCI rats. (B) Face grooming time was significantly increased in ION-CCl rats. (C) Changes in the mechanical pain threshold after injecting BMSCs around trigeminal ganglion. (D) Changes in face grooming time after injecting BMSCs around trigeminal ganglion. ${ }^{*} p<0.05,{ }^{* *} p<0.01$, $* * * p<0.001$.

significantly from the BMSC group on the 16th, 20th, and 24th days after modeling $(p<0.001)$. In the video behavior tests, face grooming time increased in both groups after modeling compared with previous modeling. After the intervention, the face grooming time of the BMSC group was less than that of the PBS group $(p<0.05)$, and it had recovered to baseline level on the 16th day after modeling $(p>0.05)$. There was no decrease in face grooming time in the PBS group, which differed significantly $(p<0.05)$ from the BMSC group on the 16th, 20th, and 24th days after modeling (Figure 4C and D).

\section{Trigeminal Ganglion Demyelination Could Be Repaired by Injecting BMSCs Around Ipsilateral Trigeminal Ganglion}

Trigeminal ganglion of rats in the ION-CCI and sham groups were removed on the 16th day after modeling. Transmission electron microscopy indicated that the ION-CCI nerve fiber structure was damaged. The thickness of the myelin sheath was uneven, and it was disintegrated and broken focally, exhibiting bead-like, curly hair-like, insect-like, or vacuolated changes. The axon was thin and off-center, and axon density was slightly uneven. Schwann cell organelles were markedly swollen, and myelin fragments were evident in the cytoplasm. In the sham group, however, the structure of nerve fibers was almost normal, the myelin sheath was dense and uniform, Schwann cell structure was clear, and the cytoplasm was uniform, except for some detachment of the axon (Figure 5).

The trigeminal ganglion of rats in the BMSC and PBS groups were removed on the 24th day after modeling. Transmission electron microscopy of trigeminal ganglion indicated that the BMSC group's nerve fiber structures had recovered significantly. The thickness of the myelin sheath was generally uniform. The lamellar structure was arranged more neatly, it was vacuolated, and curly hair-like loosening was reduced. Axon detachments were reduced, and Schwann cell organelle swelling was significantly reduced. In the PBS group, however, the trigeminal ganglion nerve fiber structure did not recover significantly. Many beaded, curly, insect-like, or vacuolated changes were still visible in the myelin sheath, and axon detachments remained. Schwann cell organelle swelling was slightly reduced (Figure 6). 
A

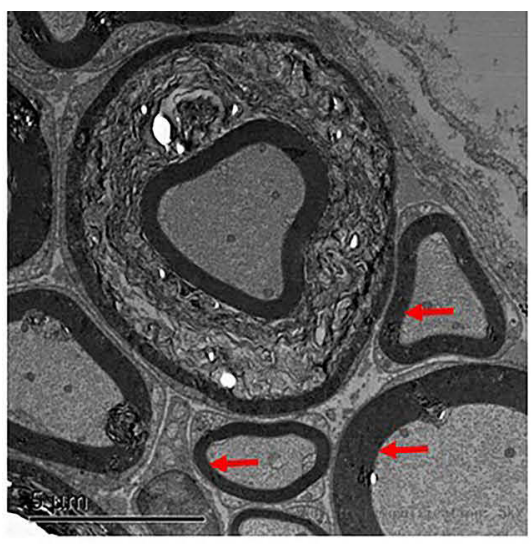

B

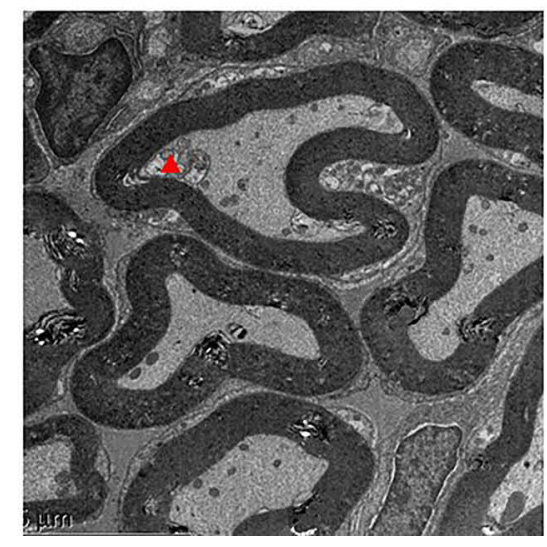

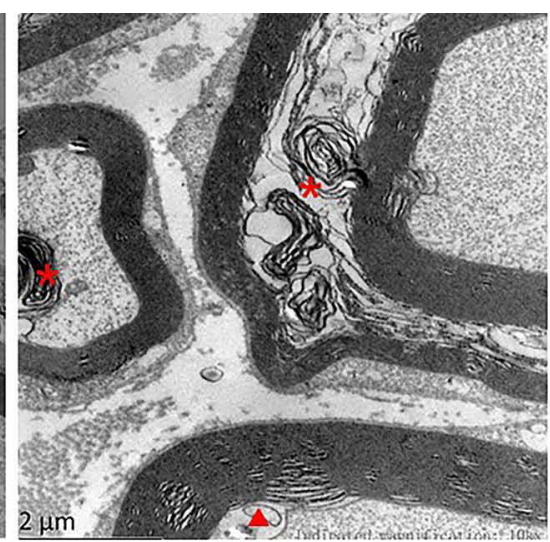
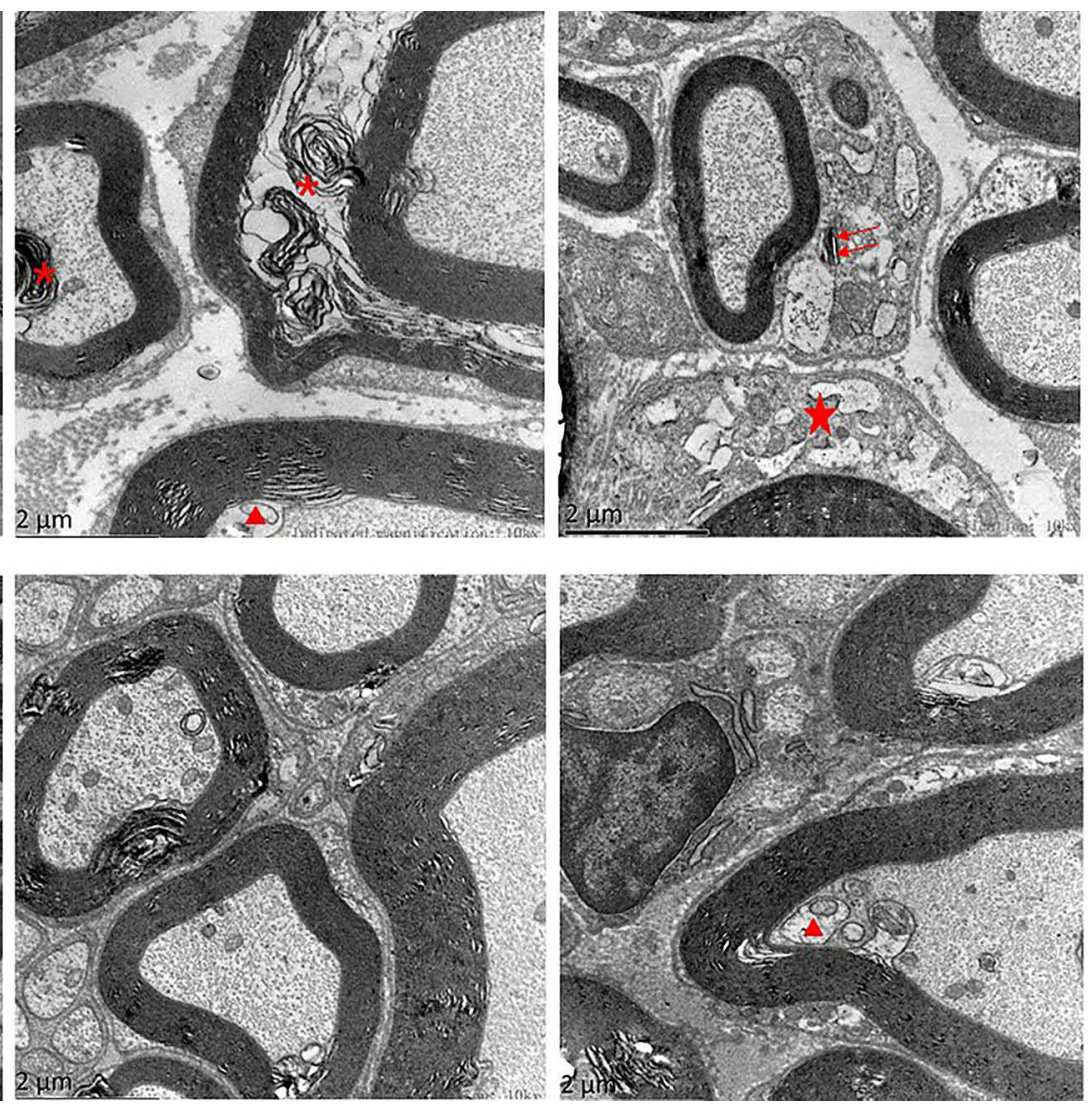

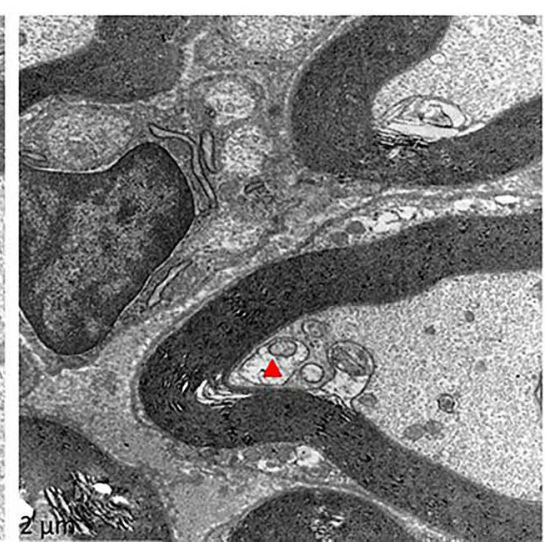

Figure 5 The trigeminal ganglion of ION-CCl rats observed via transmission electron microscopy. (A) ION-CCl group: the trigeminal ganglion exhibited varying degrees of demyelination changes. These changes included detachment of the axon (triangle), uneven thickness of the myelin sheath (arrow), vacuolization and disorganization of the myelin sheath (asterisk), and swollen Schwann cell organelles (pentagram), and myelin fragments were evident in the cytoplasm (double arrow). (B) Sham group: Trigeminal ganglion with no major changes, with the exception of some detachment of the axon (triangle).

\section{BMSC Differentiation Was Not Observed in Trigeminal Ganglion}

Trigeminal ganglion of rats in the BMSC group were removed and fixed on the 24th day after modeling, and then frozen sections were generated. Immunofluorescence staining was used to detect the expression of the Schwann cell surface markers GAFP, P75, and S-100. Red cells expressing GAFP, P75, and S-100 were evident, but green cells stained with PKH67 were not, and there were no yellow cells expressing GAFP, P75, and S-100 and exhibiting PKH67 staining (Figure 7).

\section{Discussion}

In the current study, the clinical manifestations of $\mathrm{TN}$ were simulated partially by loosely ligating rat infraorbital nerves with chromium catgut. The ION-CCI rats exhibited obvious spontaneous pain and mechanical hyperalgesia, which is similar to some clinical manifestations in TN patients. Transmission electron microscopy indicated that the trigeminal ganglion of ION-CCI rats had varying degrees of demyelination changes, which is partially consistent with surgery findings, pathological sections, and electron microscopy in TN patients. ${ }^{2}$

The main finding of the present study was that injection of BMSCs around the ipsilateral trigeminal ganglion could alleviate mechanical hyperalgesia and spontaneous pain in the infraorbital innervated area of rats after infraorbital nerve injury, and no adverse reactions were observed. When BMSCs were injected on the 9th day after modeling, the pain threshold of ION-CCI rats began to rise continuously up to the 12th day after modeling, and recovered to baseline level by the 16th day after modeling and was maintained thereafter, which is similar to results reported by Guo et al. ${ }^{12}$ In video behavior tests, the length of time spent on face grooming after BMSC injection decreased, and it recovered to baseline level on the 16th day after modeling. In transmission electron microscopy, 2 weeks after BMSC treatment, the repair 
A

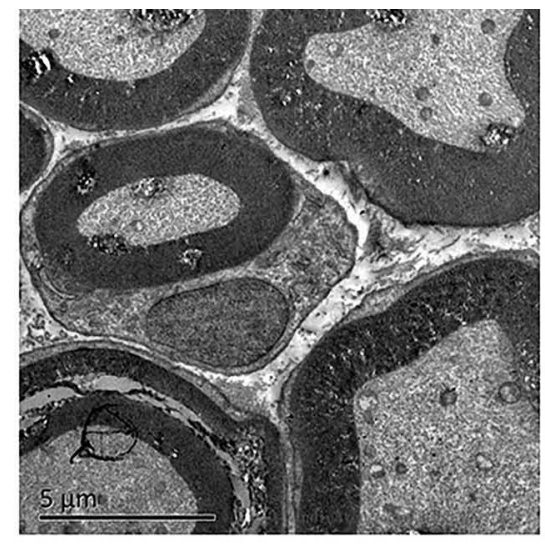

B

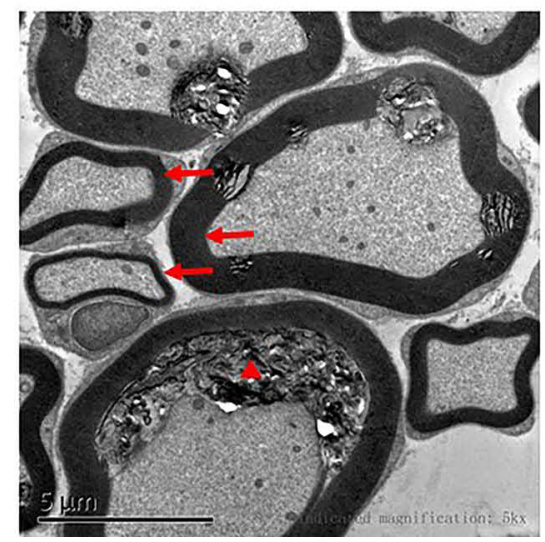

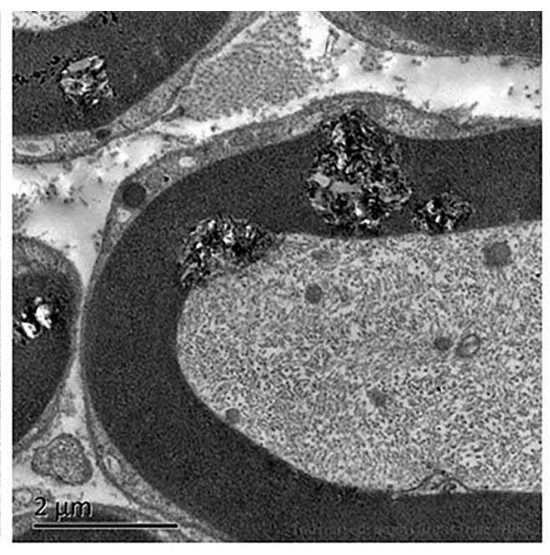
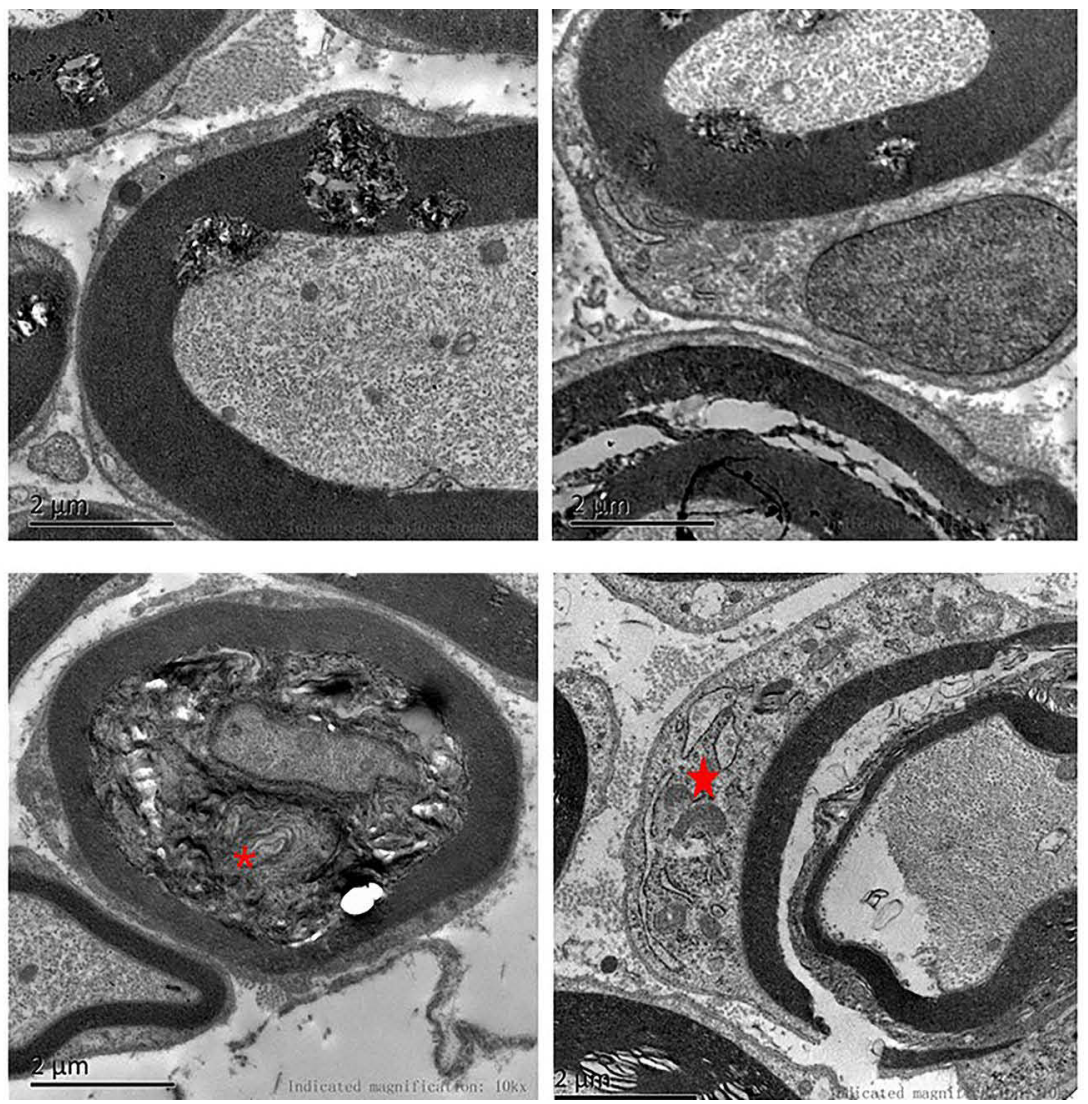

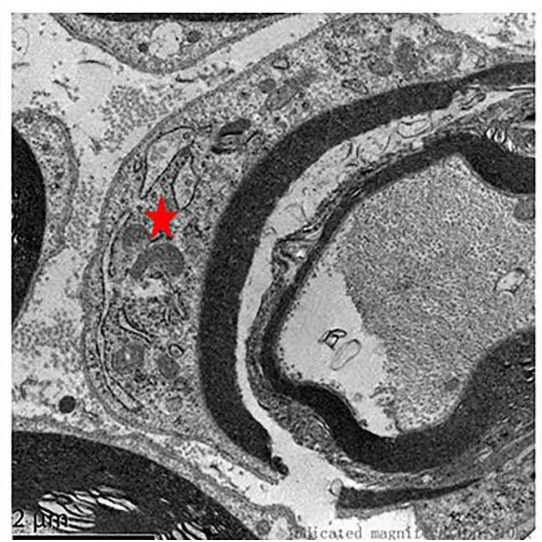

Figure 6 Recovery of trigeminal ganglion demyelination after injecting BMSCs around trigeminal ganglion. (A) BMSC group: demyelination changes in trigeminal ganglion had almost resolved. (B) PBS group: demyelination changes in trigeminal ganglion were still be evident, such as detachment of the axon (triangle), uneven thickness of the myelin sheath (arrow), vacuolization and disorganization of the myelin sheath (asterisk), and swollen Schwann cell organelles (pentagram).

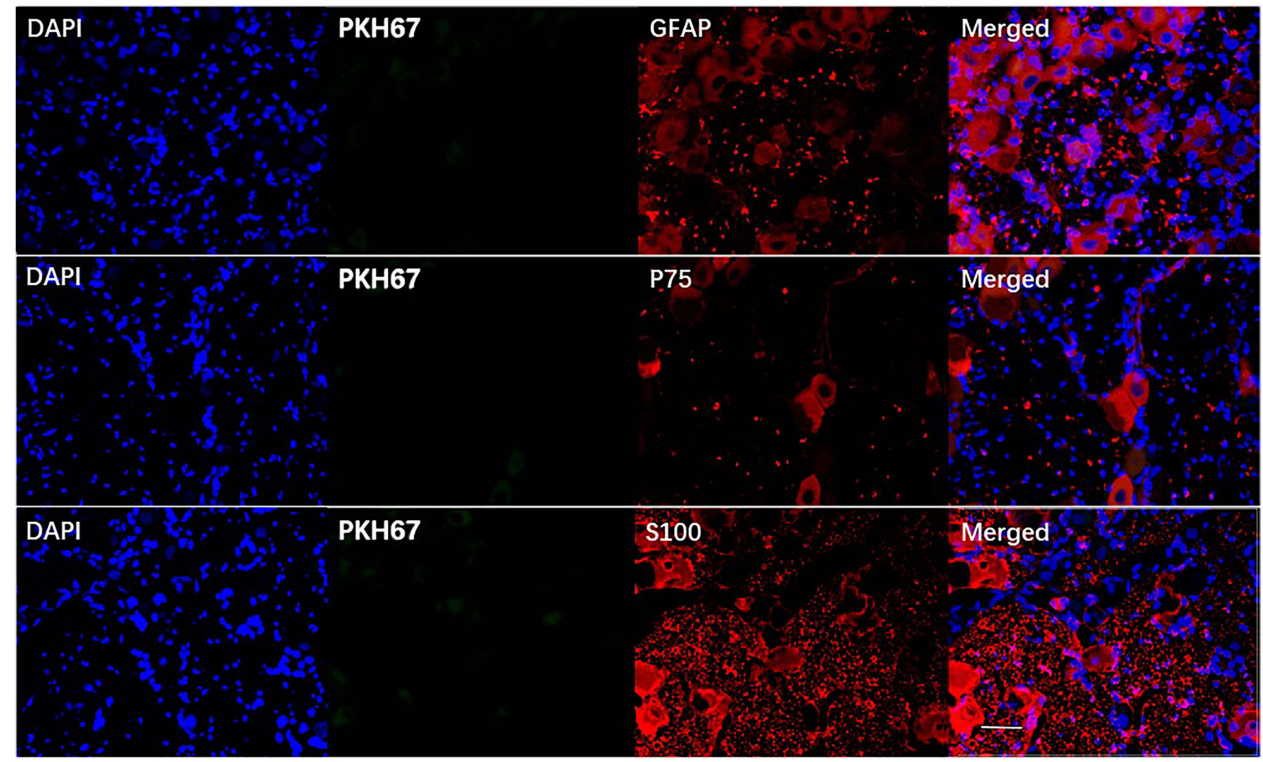

Figure 7 BMSC differentiation was not observed in trigeminal ganglion. Red indicates cells expressing GFAP, P75, and S-100, and blue indicates the nucleus. No BMSCs exhibiting green fluorescence (PKH67) were observed. Scale bar $=50 \mu \mathrm{m}$. 
effect of trigeminal ganglion demyelination was obvious, indicating that BMSCs improved the repair of TN and trigeminal nerve demyelination.

In the current study, no green-stained BMSCs were observed under fluorescence microscopy. In vitro cell cultures revealed that the fluorescence of stained BMSCs remained stable for 2 weeks, indicating that BMSCs may not colonize the trigeminal ganglion. The disappearance of BMSCs may be related to the fact that the trigeminal ganglion of Sprague Dawley rats is located at the trigeminal nerve impression in front of the tip of the petrous pyramid in the middle cranial fossa, on the ventral side and adjacent to the brain. The space between the brain and the skull is filled with cerebrospinal fluid, so BMSCs may have been injected into cerebrospinal fluid. Another possibility is that BMSCs only existed in the trigeminal ganglion tissue for a short time. Lastly, it may be that only a small number of BMSCs differentiated into Schwann cells, thus they were not observed.

Cuevas et $\mathrm{al}^{17}$ reported that in studies investigating the promotion of nerve regeneration by BMSCs only a small number of BMSCs differentiated into Schwann cells and integrated into the regenerated tissue. This indicates that the therapeutic effect of BMSCs on trigeminal nerve demyelination is not achieved via differentiation. Rather, the BMSCs may secrete substances that promote the repair of trigeminal nerve demyelination, which is consistent with the results of Ohta et al ${ }^{18}$ Ide et al, ${ }^{19}$ and Nakano et $\mathrm{al}^{20}$ in studies investigating the use of BMSCs to treat spinal cord injury in rats. In one study, cerebrospinal fluid infusion of BMSCs could treat spinal cord injury in rats. ${ }^{20}$ Only a small number of BMSCs migrated to the lesion area in rats with acute spinal cord injury, however, and they only existed for 2-7 days. No BMSCs migrated to the lesion area in rats with subacute and chronic spinal cord injury.

Mounting evidence suggests that the success of treatment of diseases by mesenchymal stem cells is due to their secretions having beneficial effects on damaged tissues. ${ }^{21-23}$ To prove that, Gama et al ${ }^{24}$ injected BMSCs and the same amount of BMSC-conditioned medium into the tail vein in a partial sciatic nerve ligation mouse model. They reported that injection of the BMSC-conditioned medium also improved the pain behavior of ION-CCI rats. The effect was similar to that of BMSC injection, and it also had a strong and long-lasting anti-injury effect. The anti-injury effect of the BMSC-conditioned medium was achieved earlier than that achieved with BMSCs. These results suggest that the therapeutic effects of BMSCs are mainly achieved via their secretions, rather than their differentiation.

BMSCs can interact with Schwann cells and other neurons via direct contact between cells, or through exosomederived bioactive mediators. ${ }^{25}$ They can reportedly secrete many proteins and exosomes with protein and functional RNA that contain a variety of nutritional factors, such as growth factors and brain-derived neurotrophic factor, glial cell linederived neurotrophic factor, and myelin basic protein, among others. These factors can regulate endogenous Schwann cell behaviors including apoptosis, migration, proliferation, and secretion, promoting myelin synthesis and nerve regeneration. They can also regulate immunity and anti-inflammatory effects and prevent immune cells near damaged nerve fibers from secreting inflammatory mediators. ${ }^{26,27}$ These therapeutic effects are evidently not dependent on their differentiation status because both differentiated and undifferentiated BMSCs reportedly exhibit beneficial molecular biological, electrophysiological, histological, and behavioral effects, ${ }^{28}$ which is consistent with the results of the current study. Although we did not observe the differentiation of BMSCs into Schwann cells, pain behavior and electron microscopy results indicated that they did have a therapeutic effect in ION-CCI rats.

\section{Conclusion}

BMSCs injected around the ipsilateral trigeminal ganglion could relieve TN in ION-CCI rats. This pain-relieving effect may be related to the repair of trigeminal ganglion demyelination. The specific mechanisms involved require further investigation.

\section{Abbreviations}

BMSCs, bone marrow mesenchymal stem cells; ION-CCI, infraorbital nerve chronic constriction injury; PBS, phosphate-buffered saline; TN, trigeminal neuralgia. 


\section{Data Sharing Statement}

The datasets generated and analyzed during the current study are available from the corresponding authors on reasonable requests.

\section{Ethics Approval}

All experimental procedures were approved by the Animal Management and Use Committee of the Department of Laboratory Animals of Central South University (approval number 2018sydw0209) and were carried out in strict accordance with the recommendations in the Guide for the Care and Use of Laboratory Animals of the National Institutes of Health.

\section{Author Contributions}

All authors made substantial contributions to conception and design, acquisition of data, or analysis and interpretation of data; took part in drafting the article or revising it critically for important intellectual content; agreed to submit to the current journal; gave final approval for the version to be published; and agreed to be accountable for all aspects of the work.

\section{Funding}

This work was supported by the National Natural Science Foundation of China (grant number 82071140) and the Hunan Provincial Natural Science Foundation of China (grant numbers 2019JJ40462 and 2019JJ40457).

\section{Disclosure}

The authors declare no conflicts of interest in this work.

\section{References}

1. Fromm GH, Terrence CF, Maroon JC. Trigeminal neuralgia current concepts regarding etiology and pathogenesis. Arch Neurol. 1984;41 (11):1204-1207. doi:10.1001/archneur.1984.04050220102026

2. Love S, Coakham HB. Trigeminal neuralgia: pathology and pathogenesis. Brain. 2001;124(12):2347-2360. doi:10.1093/brain/124.12.2347

3. Maarbjerg S, Di Stefano G, Bendtsen L, Cruccu G. Trigeminal neuralgia-diagnosis and treatment. Cephalalgia. 2017;37(7):648-657. doi:10.1177/ 0333102416687280

4. Kikkeri NS, Nagalli S. Trigeminal neuralgia. In: StatPearls [Internet]. StatPearls Publishing; 2020.

5. Devor M, Govrin-Lippmann R, Rappaport ZH. Mechanism of trigeminal neuralgia: an ultrastructural analysis of trigeminal root specimens obtained during microvascular decompression surgery. J Neurosurg. 2002;96(3):532-543. doi:10.3171/jns.2002.96.3.0532

6. Bunge MB. Bridging areas of injury in the spinal cord. Neuroscientist. 2001;7(4):325-339. doi:10.1177/107385840100700409

7. Li R, Liu Z, Pan Y, Chen L, Zhang Z, Lu L. Peripheral nerve injuries treatment: a systematic review. Cell Biochem Biophys. 2014;68(3):449-454. doi:10.1007/s12013-013-9742-1

8. Wakao S, Matsuse D, Dezawa M. Mesenchymal stem cells as a source of Schwann cells: their anticipated use in peripheral nerve regeneration. Cells Tissues Organs. 2014;200(1):31-41.

9. Kitada M. Mesenchymal cell populations: development of the induction systems for Schwann cells and neuronal cells and finding the unique stem cell population. Anat Sci Int. 2012;87(1):24-44. doi:10.1007/s12565-011-0128-4

10. Galipeau J, Sensebe L. Mesenchymal stromal cells: clinical challenges and therapeutic opportunities. Cell Stem Cell. 2018;22(6):824-833. doi:10.1016/j.stem.2018.05.004

11. Vickers ER, Karsten E, Flood J, Lilischkis R. A preliminary report on stem cell therapy for neuropathic pain in humans. $J$ Pain Res. 2014;7:255-263. doi:10.2147/JPR.S63361

12. Guo W, Wang H, Zou S, et al. Bone marrow stromal cells produce long-term pain relief in rat models of persistent pain. Stem Cells. 2011;29 (8):1294-1303. doi:10.1002/stem.667

13. Scolding NJ, Pasquini M, Reingold SC, Cohen JA. Cell-based therapeutic strategies for multiple sclerosis. Brain. 2017;140(11):2776-2796.

14. Wang H, Zhang H, Liu M, Wang N. Distal segment extracts of the degenerated rat sciatic nerve induce bone marrow stromal cells to express Schwann cell markers in vitro. Neurosci Lett. 2013;544:89-93. doi:10.1016/j.neulet.2013.03.048

15. Kernisant M, Gear RW, Jasmin L, Vit JP, Ohara PT. Chronic constriction injury of the infraorbital nerve in the rat using modified syringe needle. J Neurosci Methods. 2008;172(1):43-47. doi:10.1016/j.jneumeth.2008.04.013

16. Yang GY, Lee MK, Bae YC, et al. Intracisternal administration of COX inhibitors attenuates mechanical allodynia following compression of the trigeminal ganglion in rats. Prog Neuropsychopharmacol Biol Psychiatry. 2009;33(4):589-595. doi:10.1016/j.pnpbp.2009.02.010

17. Cuevas P, Carceller F, Garcia-Gómez I, Yan M, Dujovny M. Bone marrow stromal cell implantation for peripheral nerve repair. Neurol Res. 2004;26(2):230-232. doi:10.1179/016164104225013897

18. Ohta M, Suzuki Y, Noda T, et al. Bone marrow stromal cells infused into the cerebrospinal fluid promote functional recovery of the injured rat spinal cord with reduced cavity formation. Exp Neurol. 2004;187(2):266-278. doi:10.1016/j.expneurol.2004.01.021 
19. Ide C, Nakai Y, Nakano N, et al. Bone marrow stromal cell transplantation for treatment of sub-acute spinal cord injury in the rat. Brain Res. 2010;1332:32-47.

20. Nakano N, Nakai Y, Seo TB, et al. Effects of bone marrow stromal cell transplantation through CSF on the subacute and chronic spinal cord injury in rats. PLoS One. 2013;8(9):e73494. doi:10.1371/journal.pone.0073494

21. Cantinieaux D, Quertainmont R, Blacher S, et al. Conditioned medium from bone marrow-derived mesenchymal stem cells improves recovery after spinal cord injury in rats: an original strategy to avoid cell transplantation. PLoS One. 2013;8(8):e69515. doi:10.1371/journal.pone.0069515

22. Chuang TJ, Lin KC, Chio CC, Wang CC, Chang CP, Kuo JR. Effects of secretome obtained from normoxia-preconditioned human mesenchymal stem cells in traumatic brain injury rats. $J$ Trauma Acute Care Surg. 2012;73(5):1161-1167. doi:10.1097/TA.0b013e318265d128

23. Chang CP, Chio CC, Cheong CU, Chao CM, Cheng BC, Lin MT. Hypoxic preconditioning enhances the therapeutic potential of the secretome from cultured human mesenchymal stem cells in experimental traumatic brain injury. Clin Sci. 2013;124(3):165-176. doi:10.1042/CS20120226

24. Gama KB, Santos DS, Evangelista AF, et al. Conditioned medium of bone marrow-derived mesenchymal stromal cells as a therapeutic approach to neuropathic pain: a preclinical evaluation. Stem Cells Int. 2018;2018:8179013. doi:10.1155/2018/8179013

25. Yousefi F, Lavi Arab F, Nikkhah K, Amiri H, Mahmoudi M. Novel approaches using mesenchymal stem cells for curing peripheral nerve injuries. Life Sci. 2019;221:99-108.

26. Chen CJ, Ou YC, Liao SL, et al. Transplantation of bone marrow stromal cells for peripheral nerve repair. Exp Neurol. 2007;204(1):443-453. doi:10.1016/j.expneurol.2006.12.004

27. Wakao S, Hayashi T, Kitada M, et al. Long-term observation of auto-cell transplantation in non-human primate reveals safety and efficiency of bone marrow stromal cell-derived Schwann cells in peripheral nerve regeneration. Exp Neurol. 2010;223(2):537-547. doi:10.1016/j. expneurol.2010.01.022

28. Sayad Fathi S, Zaminy A. Stem cell therapy for nerve injury. World J Stem Cells. 2017;9(9):144-151. doi:10.4252/wjsc.v9.i9.144

Journal of Pain Research

\section{Publish your work in this journal}

The Journal of Pain Research is an international, peer reviewed, open access, online journal that welcomes laboratory and clinical findings in the fields of pain research and the prevention and management of pain. Original research, reviews, symposium reports, hypothesis formation and commentaries are all considered for publication. The manuscript management system is completely online and includes a very quick and fair peer-review system, which is all easy to use. Visit http://www.dovepress.com/testimonials.php to read real quotes from published authors.

Submit your manuscript here: https://www.dovepress.com/journal-of-pain-research-journal 